\title{
Repetition number of graphs
}

\author{
Yair Caro*and Douglas B. West ${ }^{\dagger}$ \\ Submitted: Dec 25, 2007; Accepted: Dec 22, 2008; Published: Jan 7, 2009 \\ Mathematics Subject Classification: 05C07
}

\begin{abstract}
Every $n$-vertex graph has two vertices with the same degree (if $n \geq 2$ ). In general, let $\operatorname{rep}(G)$ be the maximum multiplicity of a vertex degree in $G$. An easy counting argument yields $\operatorname{rep}(G) \geq n /(2 d-2 s+1)$, where $d$ is the average degree and $s$ is the minimum degree of $G$. Equality can hold when $2 d$ is an integer, and the bound is approximately sharp in general, even when $G$ is restricted to be a tree, maximal outerplanar graph, planar triangulation, or claw-free graph. Among large claw-free graphs, repetition number 2 is achievable, but if $G$ is an $n$-vertex line graph, then $\operatorname{rep}(G) \geq \frac{1}{4} n^{1 / 3}$. Among line graphs of trees, the minimum repetition number is $\Theta\left(n^{1 / 2}\right)$. For line graphs of maximal outerplanar graphs, trees with perfect matchings, or triangulations with 2 -factors, the lower bound is linear.
\end{abstract}

\section{Introduction}

A well-known elementary exercise states that every graph (no loops or multiedges) has two vertices with the same degree. Motivated by this, we define the repetition number of a graph $G$, written $\operatorname{rep}(G)$, to be the maximum multiplicity in the list of vertex degrees.

We study the minimum of $\operatorname{rep}(G)$ on various classes of $n$-vertex graphs. The maximum is $n$ whenever the class contains a regular graph. Some classes we study (trees, maximal outerplanar graphs, planar triangulations) have no regular graphs, but they have nearly regular graphs, allowing $\operatorname{rep}(G)$ to be as large as $n-c$ for some constant $c$. Thus we restrict our attention to minimizing $\operatorname{rep}(G)$.

Bounding $\operatorname{rep}(G)$ has structural consequences. With $\Delta(G)$ denoting the maximum vertex degree in $G$, the pigeonhole principle yields $\operatorname{rep}(G) \geq n / \Delta(G)$ (when $G$ has no isolated vertices). Equivalently, $\operatorname{rep}(G) \leq k$ implies $\Delta(G) \geq n / k$. For triangle-free graphs, this yields $\alpha(G) \geq n / k$, where $\alpha(G)$ is the independence number. Bollobás and

\footnotetext{
*Mathematics Department, University of Haifa-Oranim, Tivon 36006, Israel. email: yacaro@kvgeva.org.il

${ }^{\dagger}$ Mathematics Department, University of Illinois, Urbana, IL 61801, west@math.uiuc.edu. This research is partially supported by the National Security Agency under Award No. H98230-06-1-0065.
} 
Scott [4] answered a question of Erdős by proving that this lower bound is asympotically sharp: for $k \geq 2$, there is a triangle-free $n$-vertex graph $G$ such that $\operatorname{rep}(G) \leq k$ and $\alpha(G)=(1+o(1)) n / k$.

Another phrasing of the lower bound $\alpha(G) \geq n / k$ is that there is no sequence of triangle-free graphs (with $G_{n}$ having $n$ vertices) such that $\operatorname{rep}\left(G_{n}\right) \leq k$ and the independence number of $G_{n}$ is $o(n)$. However, Bollobás [3] showed that among $K_{4}$-free graphs with repetition number at most 5 , there is a sequence with independence number $o(n)$.

The structural consequences of making $\operatorname{rep}(G)$ small motivate studying lower bounds on $\operatorname{rep}(G)$. By a simple counting argument, we show that $\operatorname{rep}(G) \geq n /(2 d-2 s+1)$ when $G$ has $n$ vertices, average degree $d$, and minimum degree $s$. We prove that this is sharp in general by proving that certain degree lists are graphic. By explicit constructions, the bound remains sharp for trees, maximal outerplanar graphs, planar triangulations with specified minimum degree, and certain claw-free graphs.

For line graphs, the lower bound can be improved, though the growth rate of the minimum of $\operatorname{rep}(G)$ over $n$-vertex line graphs remains unknown. We prove a lower bound of $\frac{1}{4} n^{1 / 3}$ and conjecture that the truth is $\Theta\left(n^{1 / 2}\right)$. For line graphs of trees, the minimum is $\Theta\left(n^{1 / 2}\right)$. For several other classes of line graphs, we show that the repetition number is linear in the number of vertices, but the optimal coefficients are unknown.

\section{General $n$-vertex graphs}

We consider graphs having $n$ vertices, average degree $d$, and minimum degree $s$. The pigeonhole principle immediately yields $\operatorname{rep}(G) \geq n /(\Delta(G)-s+1)$. More careful counting yields a more useful lower bound.

Lemma 2.1 If $G$ is an n-vertex graph with average degree $d$ and minimum degree $s$, then $\operatorname{rep}(G) \geq\lceil n /(2 d-2 s+1)\rceil$.

Proof. Let $r=\operatorname{rep}(G)$, and define $a$ and $b$ by $n=r a+b$ with $1 \leq b \leq r$. We obtain a lower bound on the degree-sum $d n$. The $r$ smallest terms in the degree list sum to at least $r s$. The $r$ next smallest terms sum to at least $r(s+1)$. Continuing this argument shows that the degree-sum is at least the sum of $r$ copies of each value from $s$ through $s+a-1$, plus $b$ copies of $s+a$. Summing these lower bounds yields $d n \geq r a(2 s+a-1) / 2+b(s+a)$. Since

$$
r a \frac{2 s+a-1}{2}+b(s+a)=n s+\frac{n}{2}(a-1)+b \frac{a+1}{2}=n s+\frac{n}{2}\left(\frac{n}{r}-1\right)+\frac{b(r-b)}{2 r},
$$

we obtain $d n \geq n s+(n / 2)(n / r-1)$, which simplifies to $r \geq n /(2 d-2 s+1)$.

Before discussing the sharpness of Lemma 2.1 in general, we provide some explicit constructions for sharpness. The $k$ th power of a graph $G$ is the graph $G^{k}$ with vertex set $V(G)$ in which vertices are adjacent if the distance between them in $G$ is at most $k$. 
Example 2.2 Choose a positive integer $k$; in this construction $d=2 k$. Start with $C_{k p}^{k}$, which is $2 k$-regular. Group the vertices along the underlying cycle into sets of $k$ consecutive vertices. Identify each group with the $k$ vertices at one end of a copy of $P_{2 k+1}^{k}$ to form $G$.

The vertex degrees in $P_{2 k+1}^{k}$ are $k, \ldots, 2 k-1,2 k, 2 k-1, \ldots, k$ in order. The first $k$ vertices, when identified with a set $S$ in $C_{k p}^{k}$, each gain $k+1$ neighbors outside $S$ in $V\left(C_{k p}^{k}\right)$. Hence the degrees of the vertices from one copy of $P_{2 k+1}^{k}$ are $2 k+1, \ldots, 3 k$ and $2 k, 2 k-1, \ldots, k$ in the final construction. Each value from $k$ through $3 k$ occurs once. Since this holds for each copy of $P_{2 k+1}^{k}$, the degrees are uniformly distributed, with average $2 k$, $\operatorname{minimum} k$, and $\operatorname{rep}(G)=n /(4 k-2 k+1)$.

If $P_{k p}^{k}$ is the "host graph" instead of $C_{k p}^{k}$, then the resulting graph is chordal (in fact, a "k-tree"), and almost achieves equality in Lemma 2.1.

The next construction achieves sharpness for more values of $d$ but fewer values of $n$.

Example 2.3 Let $H_{p}$ be the bipartite graph with vertex set $\left\{x_{1}, \ldots, x_{p}\right\} \cup\left\{y_{1}, \ldots, y_{p}\right\}$ such that $x_{i}$ is adjacent to $y_{j}$ if and only if $i+j>p$. Note that $x_{i}$ and $y_{i}$ have degree $i$, for $1 \leq i \leq p$. This graph has sometimes been called the half-graph with $2 p$ vertices.

Given $n, d, s$ with $n, s, 2 d \in \mathbb{N}$, let $r=n /(2 d-2 s+1)$. If $r$ is an even integer and $n>2 d-s$, then we build an $n$-vertex graph $G$ with average degree $d$, minimum degree $s$, and repetition number $r$. It must have $r$ vertices of each degree from $s$ through $2 d-s$, and hence $n \geq 2 d-s+1$ is necessary. Note also that $n$ is even, since $r$ is even.

Let $F=(r / 2) H_{n / r}$; note that $F$ has $n$ vertices, with degrees 1 through $2 d-2 s+1$, equally distributed. We add to $F$ an $(s-1)$-regular graph $J$ with $V(J)=V(F)$ and $E(J) \cap E(F)=\varnothing$. Let $V_{2 i-1}$ and $V_{2 i}$ denote the partite sets of the $i$ th component of $F$.

Let $V_{1}, \ldots, V_{r}$ correspond to the vertices in a copy of $K_{r}$. Ignore $\left\{V_{2 i-1} V_{2 i}: 1 \leq i \leq\right.$ $r / 2\}$. Each of the remaining $r-2$ perfect matchings in a 1-factorization of $K_{r}$ provides up to $n / r$ disjoint perfect matchings on our vertex set by 1 -factoring copies of $K_{n / r, n / r}$. This yields a regular graph on $V(F)$ with any degree up to $(r-2) n / r$.

We may also need edges within each $V_{j}$. Since $\left|V_{j}\right|=2 d-2 s+1$ and $2 d$ may be any integer, $\left|V_{j}\right|$ may be odd or even. Regular graphs with vertex set $V_{j}$ may have even degree (when $2 d$ is even) or unrestricted degree (when $2 d$ is odd), but in either case such graphs have degree at most $2 d-2 s$.

By combining these two types of regular graphs, we can let $J$ be a regular graph on $V(F)$ with any degree up to $n-n / r-1$. Since $n-n / r-1 \geq(2 d-s+1)-(2 d-2 s+1)-1=$ $s-1$, adding $J$ can augment the degrees by the amount needed to construct a graph with the desired degree list. This proves sharpness of Lemma 2.1 in this case.

Example 2.3 does not consider odd $r$. Other explicit constructions can be given for that case. We omit these because we prove next that sharpness examples always exist. To extend the notion of sharpness when $n$ is not a multiple of $2 d-2 s+1$, we seek graphs whose degree-sum meets the refined lower bound in the counting argument for Lemma 2.1, 
including the term involving $b$. Say that a list of $a r+b$ integers from $s$ to $s+a$ is packed if it consists of $r$ copies of each value from $s$ to $s+a-1$ and $b$ copies of $s+a$ (here $1 \leq b \leq r)$

Showing that packed lists are graphic if they satisfy the obvious necessary conditions shows that in some sense the simple counting argument of Lemma 2.1 is always sharp when all graphs are allowed. We use a standard equivalent form of the Erdös-Gallai conditions characterizing graphic lists. Let $d_{1}, \ldots, d_{n}$ be a list of nonnegative integers, indexed in nonincreasing order. Erdös and Gallai [8] showed that $d$ is the degree list of some graph (no loops or multiple edges) if and only if $d$ has even sum and satisfies

$$
\sum_{i=1}^{k} d_{i} \leq k(k-1)+\sum_{i=k+1}^{n} \min \left\{k, d_{i}\right\} \quad \text { for } \quad 1 \leq k \leq n .
$$

We treat $d$ as a partition and consider the Ferrers diagram with $d_{i}$ dots in row $i$. Adding 1 for each term on the left in (1) converts the right side to a count of the first $k$ columns in the diagram. That is, the $k$ th condition is equivalent to $\sum_{i=1}^{k}\left(d_{i}+1\right) \leq \sum_{i=1}^{k} d_{i}^{*}$, where $d^{*}$ is the conjugate partition, defined by $d_{i}^{*}$ being the number of dots in the $i$ th column of the Ferrers diagram of $d$. Furthermore, if the inequalities hold for all $k$ such that $d_{k} \geq k$, then they hold for all $k$. The largest such $k$ has been denoted $\ell(d)$. We use the result in this form.

Theorem 2.4 A packed list is graphic if and only if the sum is even and ar $+b>s+a$.

Proof. The conditions are obviously necessary, since the degree sum must be even and the number of vertices must exceed the maximum degree. For sufficiency, we use the conjugate form of the Erdös-Gallai conditions as described above. Note that since $b \leq r$, the hypothesis $r a+b>s+a$ implies that $r>1$ (except for the singleton list $(0)$ ). Let $n=r a+b$.

Observe that in a packed list, successive terms differ by at most 1 . In the conjugate, they differ by $r$, except that the first $s$ terms equal $n$. Let $l=\ell(d)$, and compare $d_{l}$ and $d_{l}^{*}$. If $d_{l}+1 \leq d_{l}^{*}$, then the behavior of $d_{k}$ and $d_{k}^{*}$ as $k$ decreases implies that $d_{k}+1 \leq d_{k}^{*}$ continues to hold. Even after $d_{k}^{*}$ stabilizes (at $n$ ), the inequality still holds since $d_{1}<n$. Hence it suffices to show that $d_{l}+1 \leq d_{l}^{*}$. This will work except in one case.

By definition $d_{l} \geq l$, but also $l \geq d_{l+1} \geq d_{l}-1$. Hence $d_{l} \in\{l, l+1\}$. If $d_{l}=l+1$, then $d_{l+1}=l$, and hence $d_{l}^{*}=l+r$. Since $r>1$, we have $d_{l}+1 \leq d_{l}^{*}$ in this case.

Always $d_{l}^{*} \geq l$, since otherwise $d_{l}<l$. If $d_{l}=l$ and $d_{l}^{*}>l$, then again $d_{l}+1 \leq d_{l}^{*}$. This leaves the case $d_{l}=d_{l}^{*}=l$, where the desired inequality fails by 1 . If $l=1$, then $r=1$, which is impossible. If $l>1$, then we combine the inequalities for $k=l$ and $k=l-1$. Since $d_{l+1}<d_{l}$, we have $d_{l-1}=d_{l}$ since the list is packed. Now $d_{l-1}+d_{l}+2=2 l+2 \leq 2 l+r=d_{l}^{*}+d_{l-1}^{*}$. Again each desired condition is a sum of inequalities that do hold.

Packed lists have no "gaps"; that is, successive terms differ by at most 1. Our study of repetition number thus led to asking which gap-free lists are graphic. In [1], the authors 
prove that every gap-free list with largest term $k$, smallest term $s$, even sum, and length at least $k+\left\lceil\frac{k+s}{2 s}\right\rceil$ is graphic. Furthermore, this is sharp. That result is not strong enough to imply Theorem 2.4, which shows that a packed list with length at least $k+1$ is graphic. The result of [1] is sharp even though its threshold length is larger, because its sharpness examples have multiple copies of $k$ and $s$ but just one copy of each intermediate term. Such lists are as far from packed as gap-free lists can get. The problem of determining the multiplicities that enable a list to be graphic is studied in [5].

We next study whether Lemma 2.1 remains sharp within restricted families of graphs. We show that it is asymptotically sharp for several natural families of sparse graphs.

Theorem 2.5 Among n-vertex graphs, the bound from Lemma 2.1 is asymptotically sharp for trees, maximal outerplanar graphs, planar triangulations, and planar triangulations with minimum degree 4 or minimum degree 5 . The smallest repetition numbers in these families are asymptotic to $n / 3, n / 5, n / 7, n / 5, n / 3$, respectively.

Proof. Again let $d$ and $s$ denote the average and minimum degrees. When $G$ is a tree, $d=(2 n-2) / n<2$ and $s=1$. Hence $\operatorname{rep}(G)>n / 3$. If $G$ is formed by growing a path of two edges from each internal vertex of $P_{(n-2) / 3}$ (when $n \equiv 2 \bmod 3$ ), then $\operatorname{rep}(G)=n / 3+(4 / 3)$.

A maximal outerplanar graph $G$ has $2 n-3$ edges and minimum degree 2 . Hence $d=(4 n-6) / n<4$ and $s=2$, so $\operatorname{rep}(G)>n / 5$. The example in Figure 1 has roughly $n / 5$ vertices of each degree from 2 through 6 , with two of each degree in each vertical "strip". Each end has extra vertices of degrees 3 and 2 . Hence $\operatorname{rep}(G)=(n-4) / 5+2=n / 5+(6 / 5)$ when $n \equiv 4 \bmod 10$.

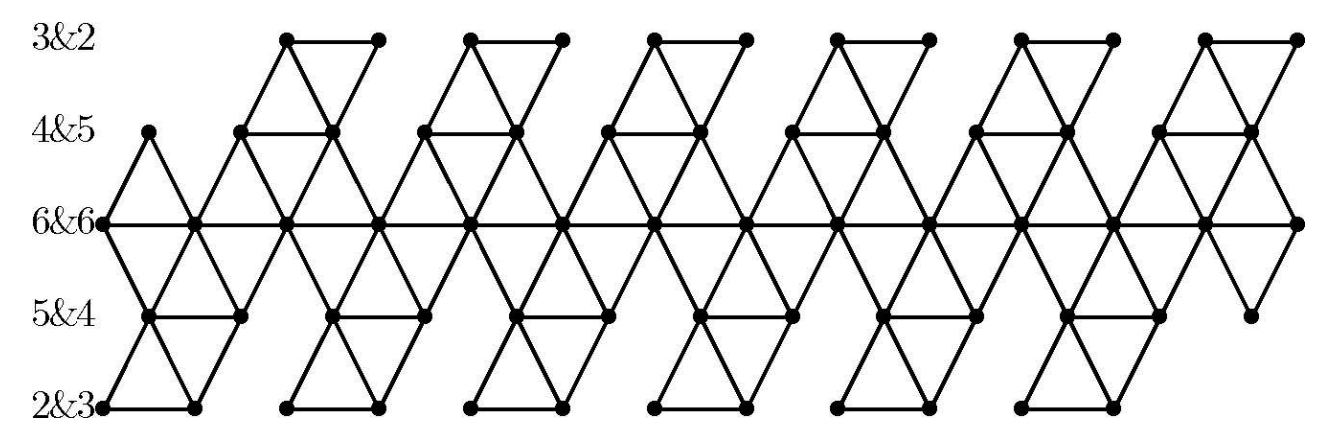

Figure 1: Maximal outerplanar graph with small repetition number.

A planar triangulation $G$ has $3 n-6$ edges and minimum degree 3 . Hence $d=(6 n-$ $12) / n<6$ and $s=3$, so $\operatorname{rep}(G)>n / 7$. We seek a repeatable tile that will assemble to a triangulation with repetition number $n / 7+O(\sqrt{n})$. We use roughly equal numbers of vertices with degrees 3 through 9 .

The vertices of the hexagonal grid have degree 3, and each lies on three hexagonal faces. We add the same triangulation inside each hexagonal face, with each vertex of the hexagon receiving two additional neighbors inside the hexagon; these vertices thus reach 
degree 9 . Inside are two vertices with each degree from 3 through 8 . Such a tile appears on the left in Figure 2, with the edges of the hexagonal grid in bold.

Since each grid vertex lies on three hexagons, the frequency of vertices with degree 9 is the same as the frequency of the other degrees when we tile the entire plane. Making the graph finite by stopping at the boundaries of hexagons reduces the degree of boundary vertices; there are $O(\sqrt{n})$ of them. Carefully triangulating the unbounded face to distribute the degrees of its vertices fairly equally reduces the difference between maximum and minimum multiplicity below $O(\sqrt{n})$, but without taking that care we still have $\operatorname{rep}(G) \leq n / 7+O(\sqrt{n})$.

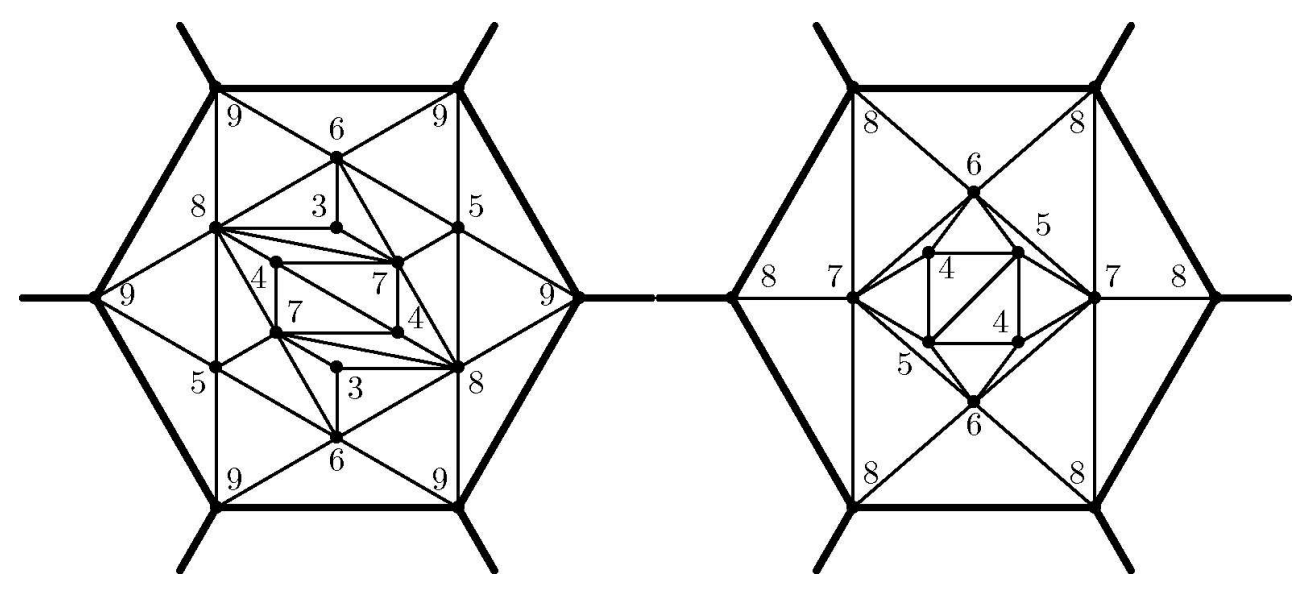

Figure 2: Tiles for planar triangulation with minimum degrees 3 and 4 .

For triangulations with minimum degree 4 we take a similar approach. Since again $d<6$, but now $s=4$, we have $\operatorname{rep}(G) \geq n / 5$. This time the vertices of the hexagonal grid have degree 8 , and within each tile we have two vertices of each degree from 4 through 7, as shown on the right in Figure 2. To reach degree 8, a grid vertex has two neighbors inside each tile where it is incident to a horizontal edge and only one neighbor inside the third tile. The analysis of the boundary is as above, and $\operatorname{rep}(G) \leq n / 5+O(\sqrt{n})$.

For triangulations with minimum degree 5, the computation with $d<6$ and $s=5$ yields $\operatorname{rep}(G) \geq n / 3$. Our construction looks somewhat different from those above. Again we use the hexagonal grid, but there are no added vertices. Group the vertices of the grid in "rows" as suggested in Figure 3. In each set of six consecutive rows, two rows have all vertices of degree 6, and the other four alternate vertices of degrees 5 and 7 .

This is achieved by inserting a path of three edges to triangulate each hexagonal tile, contributing to the degrees of the vertices in the two central rows of vertices in the strip of hexagons. When the central edges of the paths in the strip are parallel, each vertex receives two incident edges from one side and one from the other and reaches degree 6 . When the central edges alternate positive and negative slope, half of the vertices receive two incident edges and half receive four, thus creating vertices of degrees 5 and 7 . We make the central edges parallel in a third of the strips and alternating in the others. The comments on the boundary are as before. 


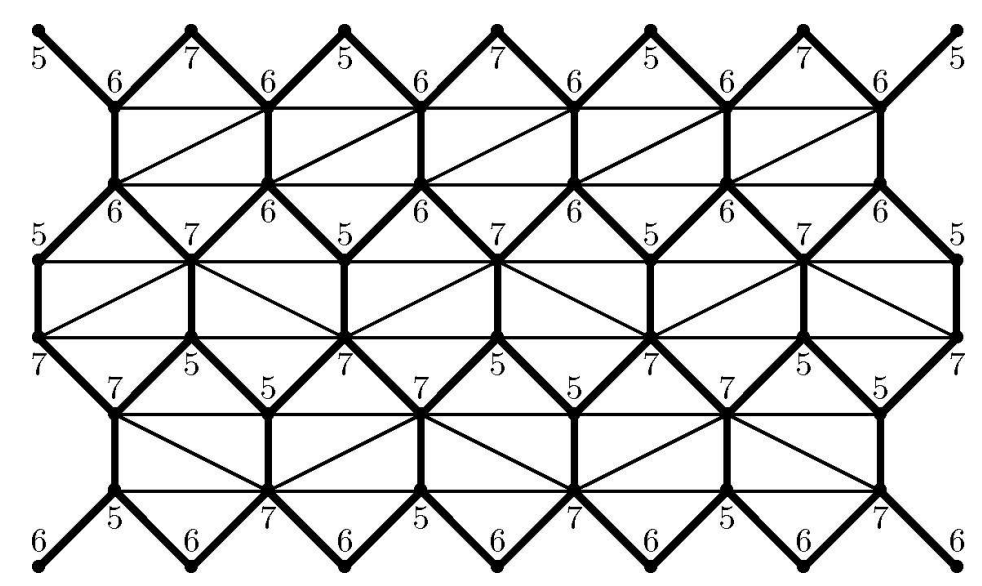

Figure 3: Planar triangulation with minimum degree 5 and low repetition.

The graphs in the constructions of Theorem 2.5 have many induced claws. The constructions of the next theorem avoid these.

Theorem 2.6 For $n, r \in \mathbb{N}$ with $2 \leq r \leq n$, there is a claw-free graph with $n$ vertices and repetition number $r$.

Proof. Let $H_{p}^{\prime}$ be the graph obtained from the half-graph $H_{p}$ by completing each partite set into a clique. Call $H_{p}^{\prime}$ the augmented half-graph. The vertex degrees in $H_{p}^{\prime}$ are $p$ through $2 p-1$, each with multiplicity 2 . Since $V\left(H_{p}^{\prime}\right)$ is the union of two cliques, $H_{p}^{\prime}$ is claw-free.

We construct an $n$-vertex claw-free graph $G_{n, r}$ with $\operatorname{rep}\left(G_{n, r}\right)=r$. Let "+" denote disjoint union of graphs. If $n-r$ is odd, then we let $G_{n, r}=G_{n-1, r}+K_{1}$, which suffices since our construction when $n-r$ is even has no isolated vertices. Hence we may assume that $n-r$ is even and let $p=(n-r) / 2$. Since $\operatorname{rep}\left(H_{p}^{\prime}\right)=2$, we may assume that $3 \leq r \leq n$.

Note that complete graphs are claw-free. Also the graph $K_{r}^{\prime}$ formed by deleting one edge from $K_{r}$ is claw-free. If $p \leq r-1 \leq 2 p-1$, then let $G=K_{r}^{\prime}+H_{p}^{\prime}$; otherwise, let $G=K_{r}+H_{p}^{\prime}$. Since $K_{r}, K_{r}^{\prime}$, and $H_{p}^{\prime}$ are all claw-free, also $G$ is claw-free. If $G=K_{r}+H_{p}^{\prime}$, then $H_{p}^{\prime}$ has no vertex of degree $r-1$, and $\operatorname{rep}(G)=r$.

If $G=K_{r}^{\prime}+H_{p}^{\prime}$, then two vertices of $H_{p}^{\prime}$ have degree $r-1$, and changing from $K_{r}$ to $K_{r}^{\prime}$ leaves $r$ vertices of degree $r-1$. Also $G$ has four vertices of degree $r-2$ if $r-1>p$ (if $r-1=p$, then degree $r-1$ enters with multiplicity 2 ).

Multiplicity 4 for $r-2$ causes no problem unless $r=3$. This case requires $(n-3) / 2=$ $p \leq r-1=2$, or $n \leq 7$. Since $n-r$ is even, it suffices to present claw-free graphs for $n=5$ and $n=7$ with repetition number 3 ; these are $K_{5}^{\prime}$ and $K_{5}^{\prime}+K_{2}$.

When $r$ is even, the graph $(r / 2) H_{p}^{\prime}$ is an instance of the construction in Example 2.3. It has $r p$ vertices, and indeed $r=n /(2 d-2 s+1)=r p /(3 p-1-2 p+1)$. Hence this class of claw-free graphs also achieves equality in the bound from Lemma 2.1. 


\section{$3 \quad$ General $m$-vertex line graphs}

Although $H_{p}^{\prime}$ is claw-free, it is not a line graph when $p \geq 3$. It is easy to show that the subgraph induced by $\left\{x_{1}, y_{1}, x_{2}, y_{2}, x_{p}, y_{p}\right\}$ is not an induced subgraph of any line graph; in fact, it is a forbidden induced subgraph in the characterization of line graphs by Beineke [2] (different for $p=3$ and $p>3$ ).

For line graphs, the lower bounds on the repetition number can be strengthened. We seek lower bounds that apply to all $m$-vertex line graphs (we use $m$ to distinguish between the numbers of vertices and edges in the graph whose line graph we study). Although general graphs with $m$ vertices may have repetition number as small as 2, and this also holds for the claw-free augmented half-graph, for line graphs the lower bound will grow with $m$.

When $2 d-2 s+1$ is small, the lower bound from Lemma 2.1 is large and can be sharp on line graphs; we present easy examples. For an edge $x y$ in a graph $G$, the degree of vertex $x y$ in $L(G)$ is $d_{G}(x)+d_{G}(y)-2$; we call it the edge-degree of $x y$ in $G$.

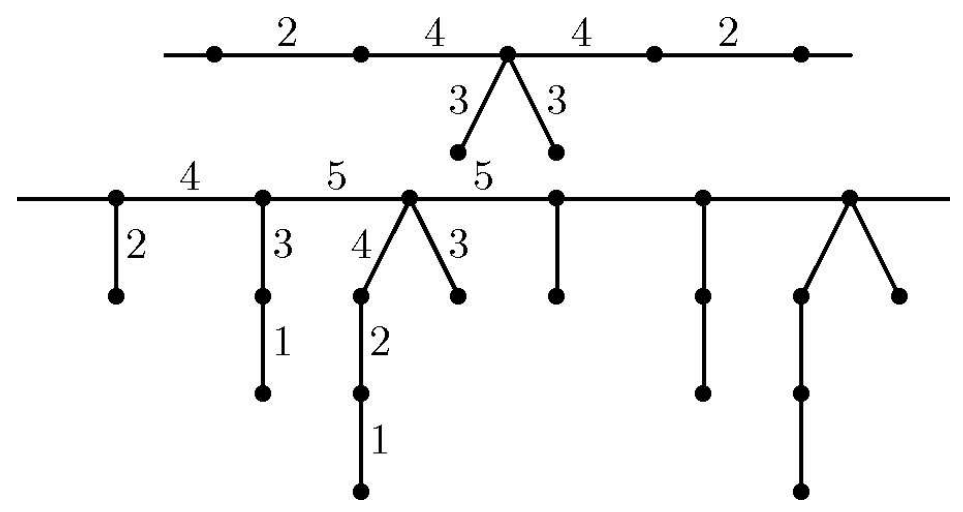

Figure 4: Unicyclic graphs with equal edge-degree multiplicities

Example 3.1 In Figure 4 we show portions of two "periodic" unicyclic graphs. In the first, the length of the cycle is a multiple of 4 . At every fourth vertex, add two pendant edges. The edge-degrees are 2,3,4 with equal multiplicity.

In the second graph, the length of the cycle is a multiple of 3 . The graphs attached at the vertices rotate among $P_{2}, P_{3}, P_{5}$, where the copies of $P_{2}$ and $P_{3}$ have an endpoint merged with the vertex on the cycle, but the copies of $P_{5}$ have a neighbor of an endpoint merged. The edge-degrees are 1,2,3,4,5 with equal multiplicity.

Since the edge-degrees are equally distributed over an interval, in the line graphs of these graphs the repetition number equals the lower bound from Lemma 2.1.

Finding connected graphs with equally distributed edge-degrees (to match the bound from Lemma 2.1) is difficult, but it is easy without the connectedness requirement, and connected examples come close. 
Proposition 3.2 For each pair $(s, d)$ with $s, 2 d \in \mathbb{N}$ and $s \leq d$, there exist arbitrarily large graphs whose edge-degrees are equally distributed over $\{s, \ldots, 2 d-s\}$. Also there are connected graphs that in addition to this "uniform" distribution have a small number of edges with larger edge-degrees.

Proof. We use components whose line graphs are regular, such as stars. Let $M$ be a multiple of each element of $\{s+1, \ldots, 2 d-s+1\}$. For $s+1 \leq j \leq 2 d-s+1$, take $M / j$ components isomorphic to $K_{1, j}$. The resulting graph has $M$ edges with each edge-degree in $\{s, \ldots, 2 d-s\}$.

To form a connected graph, use the disconnected construction for $(s-1, d-1 / 2)$ and adding one vertex $v$ adjacent to the centers of the stars. Now the edges of the original stars have the desired edge-degrees and the edges incident to $v$ have much larger edge-degrees. There are not so many such edges, and their edge-degrees have small multiplicity.

Whether connected or not, the repetition number in these constructions is linear in $m$, since $s$ and $d$ are fixed. Next we study how small it can be in terms of $m$. Let $N_{G}(v)$ denote the set of neighbors of vertex $v$ in $G$.

Theorem 3.3 If $G$ is a graph with $m$ edges, then $\operatorname{rep}(L(G)) \geq \frac{1}{4} m^{1 / 3}$.

Proof. Let $D$ be the maximum vertex degree in $G$, so that all edge-degrees are at most $2(D-1)$. Since at most $2 D-1$ distinct edge-degrees are available, $\operatorname{rep}(L(G)) \geq$ $m /(2 D-1)$. Let $a=m / D$. We consider two cases.

Case 1: $a \geq m^{1 / 3}$. Since $m=a D$, we have $\operatorname{rep}(L(G)) \geq \frac{m}{2 D-1}>\frac{a}{2} \geq \frac{1}{2} m^{1 / 3}$.

Case 2: $a \leq m^{1 / 3}$. Since $m=a D$, we have $D=m / a \geq m^{2 / 3} \geq a^{2}$, so $a \leq D^{1 / 2}$. Let $v$ be a vertex of degree $D$ in $G$. With $b_{1}, \ldots, b_{D}$ being the degrees of the vertices in $N_{G}(v)$,

$$
\sum_{i} b_{i}<2|E(G)|=2 m=2 a D \leq 2 D^{3 / 2}
$$

On the other hand, suppose that among $N_{G}(v)$, no degree appears more than $r$ times, where $r=\frac{1}{4} D^{1 / 2}$. Since $\left|N_{G}(v)\right|=D$, we obtain the smallest sum of these degrees when the list of them is packed. From the computation in Lemma 2.1, with $s=1$, we obtain $\sum b_{i} \geq D \cdot 1+(D / 2)(D / r-1)$. Thus $\sum b_{i} \geq D^{2} /(2 r)=2 D^{3 / 2}$, which contradicts (2).

We conclude that some degree appears more than $r$ times among $N_{G}(v)$. Since the edges from $v$ to $N_{G}(v)$ have a fixed endpoint, any degree that occurs more than $r$ times in $N_{G}(v)$ occurs more than $r$ times as a degree in $L(G)$. Hence $\operatorname{rep}(L(G))>r=\frac{1}{4} D^{1 / 2}$. Since in Case 2 we have $D \geq m^{2 / 3}$, we conclude that $\operatorname{rep}(L(G))>\frac{1}{4} m^{1 / 3}$.

Proposition 3.4 For infinitely many $m$ there is a graph $G$ with $m$ edges and $\operatorname{rep}(L(G)) \leq$ $\sqrt{4 m / 3}$. 
Proof. As in Proposition 3.2, we use a disjoint union of stars. Fix an integer $r$. For $1 \leq i \leq r$, include $\lfloor r / i\rfloor$ components that are stars with $i$ edges, totalling $m$ edges. At most $r$ edges have edge-degree $i-1$, for $1 \leq i \leq r$, so $\operatorname{rep}(L(G)) \leq r$.

There are at most $r$ edges in stars with $i$ edges, so $m \leq r^{2}$; we need a lower bound. For $r \geq i>r / 2$, we lose $r-i$ edges from the count of $r$ in stars of size $i$. For $r / 2 \geq i>r / 3$, we lose $r-2 i$ edges. For $r / 3 \geq i>r / 4$, we lose $r-3 i$. Summing the deficiencies yields $m \geq r^{2}-r^{2} \sum_{j=1}^{r}\left[2 j(j+1)^{2}\right]^{-1}$. After approximating the sum with an integral, $m \geq(3 / 4) r^{2}$. Thus $\operatorname{rep}(G) \leq \sqrt{4 m / 3}$.

The number of centers of the stars in Proposition 3.4 is on the order of $r \ln r$. Hence adding a single vertex adjacent to the centers yields a connected example (in fact a tree) without disturbing the asymptotic computation.

Conjecture 3.5 The minimum of $\operatorname{rep}(L(G))$ over m-edge graphs is $\Theta\left(m^{1 / 2}\right)$.

\section{Line graphs of sparse graphs}

For various families of sparse graphs with $m$ edges, such as trees, maximal outerplanar graphs, and triangulations, we can improve the lower bounds on the repetition number of the line graphs in terms of $m$. We begin with a general argument.

Theorem 4.1 Let $G$ be a graph with average degree $d$, minimum degree $s$, and $m$ edges. If $d \geq s \geq 1$, then $\operatorname{rep}(L(G)) \geq \alpha \sqrt{m}-1$, where $\alpha=s / \sqrt{c d(c d-s)}$ with $c=2 d-2 s+1$.

Proof. Since $G$ has average degree $d$, we have $m=d n / 2$, where $n=|V(G)|$. Recall that $\operatorname{rep}(G) \geq n / c$, from Lemma 2.1. Let $A$ be a set of $\operatorname{rep}(G)$ vertices in $G$ having the same degree; this degree is at least $s$.

Let $X$ be the set of edges having both endpoints in $A$. Let $Y$ be the set of edges having one endpoint in $A$. With $|A| \geq n / c$, we have $|Y| \geq s|A|-2|X| \geq n s / c-2|X|$.

All members of $X$ have the same edge-degree, so the proof is complete if $|X| \geq \alpha \sqrt{m}$. Hence we may assume that $|X|<\alpha \sqrt{m}$, so $|Y| \geq s|A|-2|X|>n s / c-2 \alpha \sqrt{m}$.

If there are more than $k$ distinct degrees among the vertices outside $A$ having neighbors in $A$, then these degrees sum to more than $k^{2} / 2$, and yet the sum is at most $d n-s|A|$. Thus $k \leq \sqrt{2 d n-2 s n / c}$. Now the edges of $Y$ have the same degree at their endpoint in $A$ and at most $\sqrt{2 d n-2 s n / c}$ distinct degrees at the other endpoint. This yields $\operatorname{rep}(L(G)) \geq|Y| / \sqrt{2 d n-2 s n / c}$.

If $\operatorname{rep}(L(G)) \leq \alpha \sqrt{m}$, then we now have

$$
\alpha \sqrt{m} \geq \frac{n s / c-2 \alpha \sqrt{m}}{\sqrt{2 d n-2 s n / c}} .
$$

Rearranging and using $m=n d / 2$ yields $\alpha \geq s /(\sqrt{c d(c d-s)}+c d / \sqrt{m})$. 
The effect of the lower-order term in the denominator is quite small. Using $s /(a+b)>$ $(s / a)(1-b / a)$ with $b=c d / \sqrt{m}$ and $a=\sqrt{c d(c d-s)}$, we obtain $\alpha \sqrt{m}>\frac{s \sqrt{m}}{c d(c d-s)}-\frac{s}{c d-s}$. The amount subtracted is less than 1 . Thus if $\alpha$ is set to $s / \sqrt{c d(c d-s)}$, then we obtain $\operatorname{rep}(L(G)) \geq \alpha \sqrt{m}-1$.

For several families of sparse graphs with a linear number of edges, Theorem 4.1 gives lower bounds that are multiples of $n^{1 / 2}$ instead of the previous $n^{1 / 3}$. With Proposition 3.4, we conclude that for line graphs of trees with $m$ edges, the asymptotic growth rate of the smallest repetition number is $\Theta\left(m^{1 / 2}\right)$.

Corollary 4.2 For a tree or a maximal planar graph with $m$ edges, the repetition number of the line graph is at least $\sqrt{m / 30}$ or at least $\sqrt{m / 182}$, respectively.

Proof. Let $n$ be the number of vertices in the graph $G$. In the notation of Theorem 4.1, in each case we compute the lower bound $s \sqrt{m} /[c d(c d-s)]-1$.

For a tree, $s=1, d=2-2 / n$, and $c=3-4 / n$ yield $\operatorname{rep}(L(G)) \geq \sqrt{m / 30}$.

For a maximal planar graph, $s=3, d=6-12 / n$, and $c=7-12 / n$ yield $\operatorname{rep}(L(G)) \geq$ $\sqrt{m / 182}$.

The computation of Corollary 4.2 also yields $\operatorname{rep}(L(G)) \geq \sqrt{m / 90}$ for a maximal outerplanar graph, but Corollary 4.5 will greatly improve that. The improvements in Corollary 4.5 for trees and maximal planar graphs are valid only for subclasses of those families.

Our final general result employs a technique of looking only at some of the edges. The approach fits into the general theme of finding "light" edges in planar graphs. The weight $w(x y)$ of an edge $x y$ in a graph $G$ is $d_{G}(x)+d_{G}(y)$. Since shifting the values in a list by 2 does not change the multiplicities, instead of studying the edge-degrees we may equivalently study the maximum multiplicity of the weights of edges of $G$. An edge is light in $G$ if its weight is bounded by a specified constant. If the sum of the weights of the light edges is large, then there must be large multiplicity of some weight.

The theory of light edges in planar graphs has many applications, particularly in coloring problems. Recent surveys of this topic can be found in [6] and [9].

Theorem 4.3 Let $Q$ be a set of $q$ edges with weight at least $s$ in a graph $G$. If the total weight of edges in $Q$ is at most $c q$, then $\operatorname{rep}(L(G)) \geq \max _{k>s} \frac{2(k-c)}{(k-s)(k-s+1)} q$.

Proof. Let $X_{i}$ be the number of edges of weight $i$ in $Q$. For any $k$ with $k>s$,

$$
c q \geq \sum_{i \geq s} i X_{i} \geq \sum_{i=s}^{k-1} i X_{i}+k \sum_{i \geq k} X_{i}=\sum_{i=s}^{k-1} i X_{i}+k\left(q-\sum_{i=s}^{k-1} X_{i}\right)=k q+\sum_{i=s}^{k-1}(i-k) X_{i} .
$$

Letting $r=\operatorname{rep}(L(G))$, each $X_{i}$ is at most $r$. With the previous computation, we obtain

$$
(k-c) q \leq \sum_{i=s}^{k-1}(k-i) X_{i} \leq r \sum_{i=s}^{k-1}(k-i),
$$


which yields $r \geq \frac{2(k-c)}{(k-s)(k-s+1)} q$.

We apply Theorem 4.3 with $Q$ being the edge set of an appropriate subgraph of $G$. We obtain a bound in terms of the order, minimum degree, and maximum degree of that subgraph, and we are particularly interested when it is a spanning regular subgraph.

Corollary 4.4 Let $G$ be an n-vertex graph with average degree $d$ and minimum degree $z$. Let $H$ be a p-vertex subgraph with minimum degree $a$ and maximum degree $b$. If every edge of $H$ has weight at least $s$ in $G$, then $\operatorname{rep}(L(G)) \geq \max _{k>s} \frac{k a p-2(b d n-(n-p) z)}{(k-s)(k-s+1)}$. If $a=b$ and $p=n$, then $\operatorname{rep}(L(G)) \geq \max _{k>s} \frac{b n(k-2 d)}{(k-s)(k-s+1)}$.

Proof. Using the notation of Theorem 4.3, let $Q=E(H)$. We have $q=|Q| \geq p a / 2$. Define $c$ by letting the total weight of the edges in $Q$ be $c q$.

When we sum the edge weights in $H$, each vertex contributes its degree (in $G$ ) at most $b$ times. Hence $c q \leq b \sum_{v \in V(H)} d_{G}(v)$. We weaken this inequality by adding the nonnegative quantity $b d_{G}(v)-z$ for each vertex $v$ outside $H$. Hence $c q \leq b \sum_{v \in V(G)} d_{G}(v)-(n-p) z=$ $b d n-(n-p) z$. With $q \geq p a / 2$, we obtain $2 k q-2 c q \geq k a p-2(b d n-(n-p) z)$. Theorem 4.3 now yields the first claim here. When $a=b$ and $p=n$, the lower bound simplifies as in the second claim.

Corollary 4.5 Let $G$ be a graph with $m$ edges. If $G$ is a tree with perfect matching, a maximal outerplanar graph, or a triangulation with a 2-factor, then $\operatorname{rep}(L(G))$ is at least $m / 6, m / 14$, or $m / 33$, respectively. The lower bound improves to $m / 27$ or $m / 15$ for triangulations having a 2-factor and minimum degree 4 or 5 , respectively.

Proof. Let $n=|V(G)|$. In each case, we apply Corollary 4.4 with $H$ and $k$ chosen appropriately. In each case $H$ is a regular spanning subgraph, so $a=b$ and $p=n$, and the simple formula $\frac{b n(k-2 d)}{(k-s)(k-s+1)}$ applies.

Let $H$ be a 1 -factor in a tree $G$. Here $(b, d, s)=(1,2-2 / n, 3)$. Choosing $k=6$, we have $\operatorname{rep}(L(G))>\frac{1 \cdot n(6-4)}{3 \cdot 4}=\frac{n}{6}>\frac{m}{6}$.

Let $H$ be the spanning cycle in a maximal outerplanar graph $G$. Here $(b, d, s)=$ $(2,4-6 / n, 5)$. With $k=12$, we have $\operatorname{rep}(L(G))>\frac{2 n(12-8)}{7 \cdot 8}=\frac{n}{7}>\frac{m}{14}$.

Let $H$ be a 2-factor in a maximal planar graph $G$. We have $\operatorname{rep}(G)=m$ if $n \leq 4$. If $n \geq 5$, then $G$ is 3 -connected and cannot have adjacent vertices of degree 3 (their common neighbors would form a separating 2 -set). This yields $s \geq 7$ and $(b, d, s)=(2,6-12 / n, 7)$. With $k=18$, we have $\operatorname{rep}(L(G))>\frac{2 n(18-12)}{11 \cdot 12}=\frac{n}{11}>\frac{m}{33}$. If $G$ is restricted to minimum degree 4 , then $s \geq 8$, and choosing $k=16$ yields $\operatorname{rep}(L(G))>\frac{2 n(16-12)}{8 \cdot 9}=\frac{n}{9}>\frac{m}{27}$. If $G$ is restricted to minimum degree 5 , then $s \geq 10$, and choosing $k=14$ yields $\operatorname{rep}(L(G))>$ $\frac{2 n(14-12)}{4 \cdot 5}=\frac{n}{5}>\frac{m}{15}$.

For trees with 1-factors, this bound is fairly good. 
Example 4.6 Let $T$ be the tree obtained from $P_{7}$ by adding one pendant edge at each of the three most central vertices. Take many disjoint copies of $T$, plus one edge $x y$ disjoint from them. Make $x$ adjacent to one 3-valent vertex other than the center in each copy of $T$. Since $T$ has a perfect matching, the full tree $G$ also has a perfect matching.

In each set of 10 edges (a copy of $T$ plus the edge to $x$ ), the edge weights are two each of $3,4,5,6$, plus one 7 and one $5+(m-1) / 10$. Also $x y$ has weight $(m-1) / 10+2$. Hence $\operatorname{rep}(G)=(m-1) / 5$, close to the lower bound from Corollary 4.5 .

Question 4.7 What is the infimum of $\frac{\operatorname{rep}(L(G))}{|E(G)|}$ for the classes of trees with perfect matchings, maximal outerplanar graphs, and triangulations with 2-factors? Our constructions are at most twice the lower bounds (better for trees).

Every triangulation is 3-connected. Whitney [11] proved that 4-connected triangulations are Hamiltonian, so Corollary 4.5 applies to them. For triangulations that are not 4-connected, we obtain a weaker bound using another result about planar graphs. A 2,3-factor in a graph is a spanning subgraph in which every vertex has degree 2 or 3 . Enomoto, Iida, and Ota [7] proved that every 3-connected planar graph with minimum degree at least 4 has a connected 2,3-factor.

Corollary 4.8 Let $G$ be a triangulation with $m$ edges. If $G$ has minimum degree at least 4, then $\operatorname{rep}(L(G)) \geq m / 68$. If $G$ has minimum degree at least 5 , then $\operatorname{rep}(L(G)) \geq m / 51$.

Proof. Let $n=|V(G)|$, so $m=3 n-6$. Let $H$ be a 2,3-factor in $G$. In the main statement of Corollary 4.4 , set $(a, b, p, d, s)=(2,3, n, 6-12 / n, 8)$, where $s \geq 8$ because $G$ has minimum degree 4 . Using $k=24$, we find $\operatorname{rep}(L(G)) \geq \frac{24 \cdot 2 n-2(3 \cdot 6 n)}{16 \cdot 17}=\frac{3 n}{68}>\frac{m}{68}$. When $G$ has minimum degree 5 , set $s=10$. Now $k=26$ yields $\operatorname{rep}(L(G)) \geq \frac{26 \cdot 2 n-2(3 \cdot 6 n)}{16 \cdot 17}=\frac{n}{17}>\frac{m}{51}$.

Our last result uses another classical structural result in graph theory. Sumner [10] proved that every claw-free graph of even order has a 1-factor.

Corollary 4.9 Let $G$ be a claw-free graph with $n$ vertices, $m$ edges, average degree $d$, and minimum degree $z$. If $n$ is even, then $\operatorname{rep}(L(G)) \geq \max _{k>2 z} \frac{2 k / d-4}{(k-2 z)(k-2 z+1)} m$. If $n$ is odd, then $\operatorname{rep}(L(G)) \geq \max _{k>2 z} \frac{2 k / d-4-(k-2 z) / m}{(k-2 z)(k-2 z+1)} m$.

Proof. If $G$ has even order, then by Sumner's result $G$ has a 1 -factor $H$. If $G$ has odd order, then let $H$ be a 1 -factor in a graph obtained by deleting one vertex from $G$. In Corollary 4.4 , we set $a=b=1$ and $s=2 z$.

If $n$ is even, then $p=n$ and $\operatorname{rep}(L(G)) \geq \max _{k} \frac{k n-2 d n}{(k-2 z)(k-2 z+1)}=\max _{k} \frac{2 k / d-4}{(k-2 z)(k-2 z+1)} m$. If $n$ is odd, then $p=n-1$ and $\operatorname{rep}(L(G)) \geq \max _{k} \frac{k(n-1)-2(d n-z)}{(k-2 z)(k-2 z+1)}=\max _{k} \frac{2 k / d-4-(k-2 z) / m}{(k-2 z)(k-2 z+1)} m$. In each case the maximization is over $k>2 z$. 


\section{References}

[1] M. Barrus, S. G. Hartke, K. F. Jao, and D. B. West, Length thresholds for graphic lists with fixed maximum and minimum entries, preprint.

[2] L. W. Beineke, Derived graphs and digraphs, Beiträge zur Graphentheorie (Teubner, 1968), 17-33.

[3] B. Bollobás, Degree multiplicities and independent sets in $K_{4}$-free graphs, Discrete Math. 158 (1996), 27-35.

[4] B. Bollobás and A. D. Scott, Independent sets and repeated degrees, Discrete Math. 170 (1997), 41-49.

[5] G. Chen, J. Hutchinson, W. Piotkowski, W. Shreve, and B. Wei, Degree sequences with repeated values, Ars. Combin. 59 (2001), 33-44.

[6] R. Cole, U. Kowalik, and R. Škrekovski, A Generalization of Kotzig's Theorem and Its Application, SIAM J. Discrete Mathematics, 21 (2007), 93-106.

[7] H. Enomoto, T. Iida, and K. Ota, Connected spanning subgraphs of 3-connected planar graphs, J. Combin. Theory Ser. B 68 (1996), 314-323.

[8] P. Erdős and T. Gallai, Graphs with prescribed degrees of vertices, (Hungarian), Mat. Lapok 11 (1960), 264-274.

[9] S. Jendrol' and H.-J. Voss, A survey of light subgraphs, Discrete Mathematics, to appear.

[10] D. P. Sumner, Graphs with 1-factors. Proc. Amer. Math. Soc. 42 (1974), 8-12.

[11] H. Whitney, A theorem on graphs, Ann. of Math. (2) 32 (1931), 378-390. 